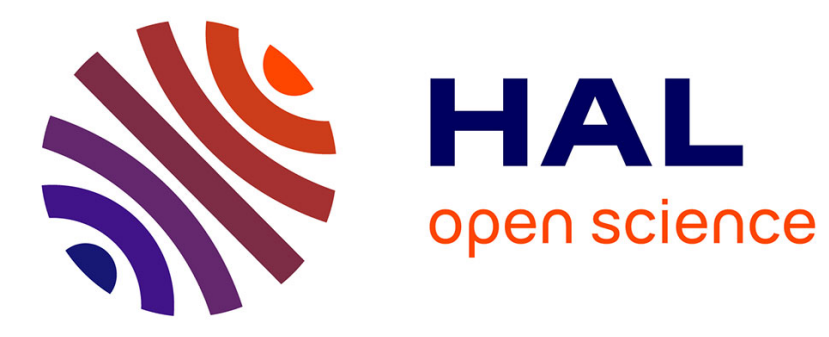

\title{
Two-sided exit problems in the ordered risk model Pierre-Olivier Goffard
}

\section{To cite this version:}

Pierre-Olivier Goffard. Two-sided exit problems in the ordered risk model. Methodology and Computing in Applied Probability, 2019, 21 (2), pp.539-549. 10.1007/s11009-017-9606-z . hal-01528204v2

\section{HAL Id: hal-01528204 \\ https://hal.science/hal-01528204v2}

Submitted on 2 Nov 2017

HAL is a multi-disciplinary open access archive for the deposit and dissemination of scientific research documents, whether they are published or not. The documents may come from teaching and research institutions in France or abroad, or from public or private research centers.
L'archive ouverte pluridisciplinaire $\mathbf{H A L}$, est destinée au dépôt et à la diffusion de documents scientifiques de niveau recherche, publiés ou non, émanant des établissements d'enseignement et de recherche français ou étrangers, des laboratoires publics ou privés. 


\title{
Two-sided exit problems in the ordered risk model
}

\author{
Pierre-Olivier Goffard*1 \\ ${ }^{1}$ Department of Statistics and Applied Probability, University of California, Santa Barbara, USA
}

November 2, 2017

\begin{abstract}
The insurance risk model in the presence of two horizontal absorbing barriers is considered. The lower barrier is the usual ruin barrier while the upper one corresponds to the dividend barrier. The distribution of two first-exit times of the risk process from the strip between the two horizontal lines is under study. The claim arrival process is governed by an Order Statistic Point Process (OSPP) which enables the derivation of formulas in terms of the joint distribution of the order statistics of a sample of uniform random variables.
\end{abstract}

MSC 2010: 60G55, 60G40, 12E10.

Keywords: Order statistic property;Joint distribution of order statistics; Finite-time ruin probabilities; First-exit time; Risk theory.

\section{Introduction}

In this work, a risk reserve process is trapped in a region delimited by two horizontal boundaries. The lower boundary represents the ruin barrier, a critical threshold that, when reached, means bankruptcy for the insurance company or the payment of insolvency penalties. The upper boundary is a target or threshold for a dividend policy. The survival function of the first-exit time of the process from the strip between the two boundaries is derived. A formula for the PDF of the first-exit time via the lower boundary is also proposed.

The ordered risk reserve process is considered. The surplus of a non-life insurance company is modeled by a stochastic process $\{R(t), t \geq 0\}$ following the dynamic

$$
R(t)=u+h(t)-\sum_{k=1}^{N(t)} U_{k}, t \geq 0 .
$$

The insurance company holds a capital $R(0)=u \geq 0$ at $t=0$ in order to avoid early ruin. The capital increases as the premiums flow at a rate $h(t)$, where $h(t)$ is a non-decreasing

*goffard@pstat.ucsb.edu 
function that satisfies $h(0)=0$ and $\lim _{t \rightarrow+\infty} h(t)=+\infty$. The number of claims up to time $t \geq 0$ is modeled through an Order Statistic Point Process (OSPP) $\{N(t), t \geq 0\}$. To each claim is associated a loss for the insurance company. These losses form a sequence $\left\{U_{k}, k \geq 1\right\}$ made of i.i.d., non-negative random variables. The claim process arrival is assumed to have the order statistic property. Namely, given that $N(t)=n$, the successive jump times $\left(T_{1}, \ldots, T_{n}\right)$ are distributed as the order statistics of $n$ i.i.d. random variables with distribution function $F_{t}(s)$, for $0 \leq s \leq t$. The OSPP family includes the homogeneous Poisson process, the linear death process and the linear birth process with immigration. Several authors have characterized the class of OSSPs under certain conditions. The reader is referred to Crump [3] and Puri [19]. Those processes have been used to model claim frequencies in various works of Willmot [23], De Vylder and Goovaert [21, 22], Lefèvre and Picard [12, 13, 14], Dimitrova et al. [6], Ignatov and Kaishev [11] and Goffard and Lefèvre [9].

This work focuses on the distribution of two first-exit times whose definition requires the introduction of the first-crossing time of the process $\{R(t), t \geq 0\}$ through the lower barrier, also known as ruin time, $\tau_{u}=\inf \{t \geq 0 ; R(t)<0 \mid R(0)=u\}$ and the first meeting time of the process with the upper barrier, $\tau_{b}=\inf \{t \geq 0 ; R(t) \geq b \mid R(0)=u\}$, where $b$ is the level of wealth the insurance company hopes to achieve. A formula is proposed for the finite-time non-ruin probability in the presence of two absorbing barriers, given by

$$
\phi(u, b, t)=\mathbb{P}\left(\tau_{u, b}>t\right) .
$$

Formula (1) corresponds to the survival function of the first-exit time of the risk process defined as

$$
\begin{aligned}
\tau_{u, b} & =\inf \{t \geq 0 ; R(t) \notin(0, b) \mid R(0)=u\} \\
& =\min \left(\tau_{u}, \tau_{b}\right) .
\end{aligned}
$$

The upper barrier is often assumed to be reflecting instead of absorbing in the study of ruin problems linked to the payment of dividends to the shareholders when a barrier strategy is applied, as in the survey of Avanzi [1] and the references therein. Nevertheless, an interpretation has been given by Dickson and Gray [5] for an upper absorbing barrier. The authors explain that if the capital of the insurance company exceeds some prescribed level, $b$, then some adjustments should be made regarding the premiums rate as it is too high to be competitive. The first-exit time (2) for processes with dynamic similar to $\{R(t), t \geq 0\}$ relates to the two-sided crossing problem of a pure jump stochastic process trapped between two paralell boundaries. Those excursion problems have received some treatment in various work, not necessarilly linked to risk theory. See, e.g., Lehmann [15], Perry et al. [17], Xu [24], Goffard and Lefèvre [10], and Dimitrova et al. [7]. A polynomial based formula is derived for the latter probability in the same vein as in Lefèvre and Picard [12].

It is of interest to know precisely the instant at which the risk process hits the upper barrier before crossing the ruin barrier. This paper also provides a formula to study the distribution of the stopping time

$$
\tau_{u, b}^{*}=\inf \{t \geq 0 ; R(t) \geq b \text { and } R(s) \geq 0 \text { for } s \leq t \mid R(0)=u\},
$$


which corresponds to the the first time at which the process reaches the level $b$ without ever being negative. From the perspective of boundary crossing, the random variable $\tau_{u, b}^{*}$ is the time at which the process escapes the region by passing through the upper boundary. A formula for the density of $\tau_{u, b}^{*}$ involving the same components as for the finite time non-ruin probability (1) is proposed. The formula ressembles the one derived in an early work of Picard and Lefèvre [18] tackling the first-meeting problem of the risk process and an upper barrier. The stopping time (3) is further called win-first exit time in reference to the win-first probability studied, for instance, in the work of Rullière and Loisel [20]. The win-first probability relates to the stopping time (3) as

$$
\mathrm{WF}(u, b)=\mathbb{P}\left(\tau_{u, b}^{*}<\infty\right) .
$$

The paper is organized as follows. Section 2 introduces the rectangular probabilities relative to the order statistics of a sample of standard uniform random variables, Section 3 gives an expression of the finite-time non ruin probability in the presence of two absorbing barrier, and Section 4 gives a formula for the density of the win-first time $\tau_{u, b}^{*}$.

\section{The joint distribution of order statistics and their polynomial representations}

Let $U=\left\{u_{i}, i \geq 1\right\}$ and $V=\left\{v_{i}, i \geq 1\right\}$ be two sequences of non-decreasing real numbers such that $0 \leq u_{1} \leq \ldots \leq u_{n} \leq 1,0 \leq v_{1} \leq \ldots \leq v_{n} \leq 1$ and $u_{1} \leq v_{1}, \ldots, u_{n} \leq v_{n}$. Let $\left(U_{1: n}, \ldots, U_{n: n}\right)$ be the order statistics of a sample of $n$ uniform random variables on $(0,1)$, later called standard uniform variables. The joint distribution of interest is described by a rectangular probability of the form

$$
\begin{aligned}
d_{n}(U, V) & =P\left[u_{1} \leq U_{1: n} \leq v_{1}, \ldots, u_{n} \leq U_{n: n} \leq v_{n}\right] \\
& =n ! \int_{u_{n}}^{v_{n}}\left[\int_{u_{n-1}}^{v_{n-1} \wedge y_{n}} \mathrm{~d} y_{n-1} \ldots \int_{u_{1}}^{v_{1} \wedge y_{2}} \mathrm{~d} y_{1}\right] \mathrm{d} y_{n} .
\end{aligned}
$$

By varying the sample size from 1 to $n$ of $U_{1}, \ldots, U_{n}$, a polynomial structure is exhibited as seen in the following result.

Proposition 1. For $u_{n} \leq x \leq 1$,

$$
P\left[u_{1} \leq U_{1: n} \leq v_{1}, \ldots, u_{n} \leq U_{n: n} \leq v_{n} \wedge x\right]=\sum_{k=0}^{n}\left(\begin{array}{l}
n \\
k
\end{array}\right)\left(v_{k+1}-x\right)_{+}^{n-k}(-1)^{n-k} d_{k} .
$$

For instance, suppose that $u_{n} \leq v_{n-m}$ for some $m \geq 1$. Consider the intervals $\left[v_{n-i}, v_{n-i+1}\right]$ for $i=0, \ldots, m$, with $v_{n+1} \equiv 1$. For $v_{n-i} \leq x \leq v_{n-i+1}$, (4) reduces to

$$
P\left[u_{1} \leq U_{1: n} \leq v_{1}, \ldots, u_{n} \leq U_{n: n} \leq v_{n} \wedge x\right]=\sum_{k=n-i}^{n}\left(\begin{array}{l}
n \\
k
\end{array}\right)\left(x-v_{k+1}\right)^{n-k} d_{k},
$$

which is a polynomial in $x$ of degree $i$. Thus, for $u_{n} \leq x \leq 1$, (4) shows that the desired probability is a piecewise polynomial function. It has been named of Sheffer type by Niederhausen [16]. Now, consider $x=u_{n}$ in (4), in which case the probability is 0 . The following result follows immediately. 
Proposition 2. Let $d_{0}(U, V)=1$. The probabilities $d_{n}(U, V)$ can be computed through the recursion

$$
d_{n}(U, V)=-\sum_{k=0}^{n-1}\left(\begin{array}{l}
n \\
k
\end{array}\right)\left(v_{k+1}-u_{n}\right)_{+}^{n-k}(-1)^{n-k} d_{k}(U, V), \quad n \geq 1 .
$$

This recursion (5) is particularly useful for producing numerical illustrations. For further reading on the link between families of polynomials and the joint distribution of order statistics, the reader is invited to consult the paper of Denuit et al. [4].

\section{The two-sided finite-time non-ruin probability}

In this section, a formula for the finite-time non-ruin probability in the case of two absorbing boundaries is derived. Recall that the risk reserve process $\{R(t), t \geq 0\}$ is defined as

$$
R(t)=u+h(t)-\sum_{k=1}^{N(t)} U_{k} \doteq u+h(t)-S_{N(t)}, t \geq 0,
$$

where $u \geq 0$ is the initial reserves. The premium is collected at a rate $h(t)$ which is a non-negative non-decreasing function that may admit discontinuities to account for the possibility of lump sum payments such as capital injections. The function $h(t)$ also satisfies $\lim _{t \rightarrow+\infty} h(t)=+\infty$ and $h(0)=0$. Its generalized inverse function is defined by $h^{-1}(x)=\inf \{t \geq 0 ; h(t) \geq x\}$ for $x \geq 0$. The claim sizes form a sequence of random variables $\left\{U_{k}, k \geq 1\right\}$ that may be governed by any joint distributions. The claim arrival process $\{N(t), t \geq 0\}$ is assumed to have the order statistic property. We denote by $\left\{S_{n}=\sum_{k=1}^{n} U_{k}, k \geq 1\right\}$ the sequence of aggregated claim sizes with $S_{0}=0$ as a convention. The process $\left\{S_{N(t)}, t \geq 0\right\}$ represents the liability of the insurance company up to time $t \geq 0$. We consider the stopping time defined by

$$
\tau_{u, b}=\inf \{t \geq 0 ; R(t) \notin(0, b) \mid R(0)=u\},
$$

and the following result provides a formula for the two-sided finite-time non-ruin probability $\phi(u, b, t)=\mathbb{P}\left(\tau_{u, b}>t\right)$.

Proposition 3. The finite-time non-ruin probability in the presence of two absorbing boundaries within the ordered insurance risk model is given by

$$
\begin{aligned}
\phi(u, b, t) & =\mathbb{E}\left[d_{N(t)}\left(\left\{\left(F_{t}\left\{h^{-1}\left[\left(S_{k}-u\right)_{+}\right]\right\}, F_{t}\left[h^{-1}\left(S_{k-1}+b-u\right)\right]\right), 1 \leq k \leq N(t)\right\}\right)\right. \\
& \left.\times \mathbb{I}_{\left\{h(t)-(b-u) \leq S_{N(t)} \leq u+h(t)\right\}}\right]
\end{aligned}
$$

where $d_{n}(\{\ldots\})$ are the rectangular probabilities as defined in Section 2 .

Proof. The stopping time $\tau_{u, b}=\inf \{t \geq 0 ; R(t) \notin(0, b) \mid R(0)=u\}$ may be rewritten as

$$
\begin{aligned}
\tau_{u, b} & =\inf \{t \geq 0 ; R(t)<0 \text { or } R(t)>b \mid R(0)=u\} \\
& =\inf \left\{t \geq 0 ; u+h(t)-\sum_{k=0}^{N(t)} U_{k}<0 \text { or } u+h(t)-\sum_{k=0}^{N(t)} U_{k}>b\right\}
\end{aligned}
$$




$$
=\inf \left\{t \geq 0 ; \sum_{k=0}^{N(t)} U_{k}<-(b-u)+h(t) \text { or } \sum_{k=0}^{N(t)} U_{k}>u+h(t)\right\} .
$$

The initial two-sided ruin problem translates into a two-sided boundary crossing problem. The ruin time corresponds to the time of first excursion of the stochastic process $\left\{S_{N(t)}=\sum_{k=0}^{N(t)} U_{k}, t \geq 0\right\}$ from the region delimited by the lower boundaries $-(b-u)+$ $h(t)$, and the upper boundary $u+h(t)$. Figure 1 displays a visualization of the boundary crossing problem and also provides a reminder of the notation. Conditioning over the

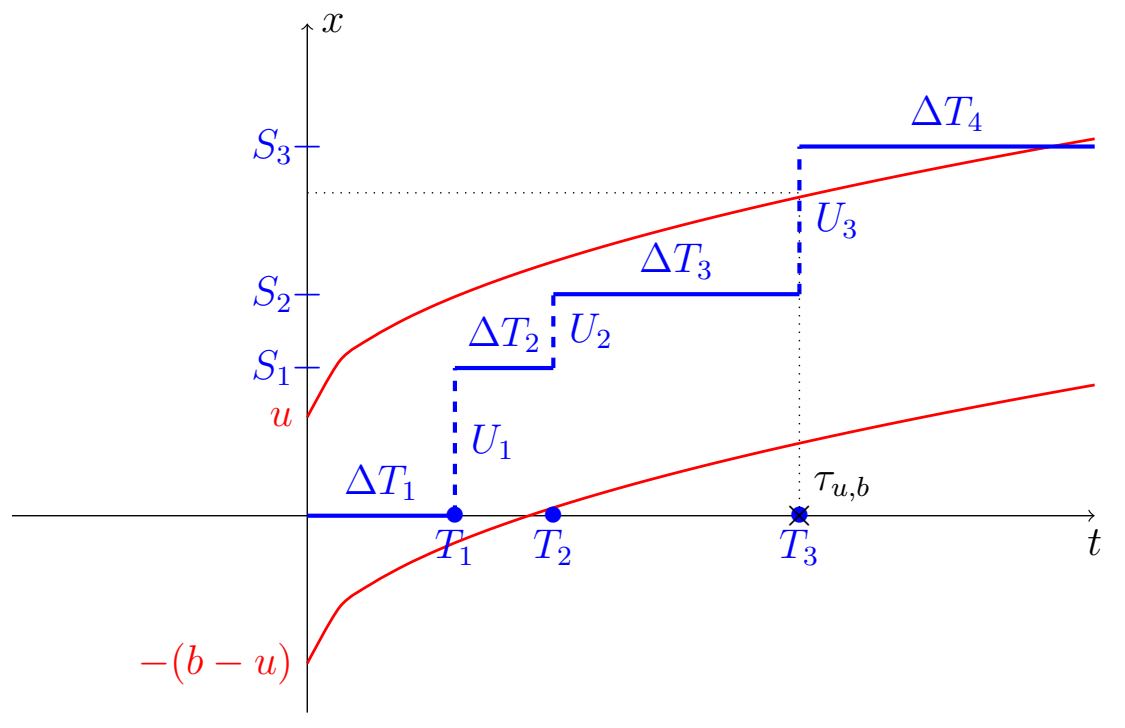

Figure 1: Ruin time in the insurance risk model. (solid,red) parallel boundaries $u+h(t)$ and $-(b-u)+h(t)$. (dashed,blue) aggregated claim amounts process.

values of $\{N(t) ; t \geq 0\}$ yields

$$
\begin{aligned}
\left\{\tau_{u, b}>t\right\}= & \bigcup_{n=0}^{+\infty}\{N(t)=n\} \cap\left\{\tau_{u, b}>t\right\} \\
= & \bigcup_{n=0}^{+\infty}\{N(t)=n\} \bigcap_{k=1}^{n}\left\{h\left(T_{k}\right)-(b-u) \leq S_{k-1}\right\} \cap\left\{h\left(T_{k}\right)+u \geq S_{k}\right\} \\
& \bigcap\left\{h(t)-(b-u)<S_{n}<h(t)+u\right\} \\
= & \bigcup_{n=0}^{+\infty}\{N(t)=n\} \bigcap_{k=1}^{n}\left\{h^{-1}\left[\left(S_{k}-u\right)_{+}\right] \leq T_{k} \leq h^{-1}\left(S_{k-1}+b-u\right)\right\} \\
& \bigcap\left\{h(t)-(b-u)<S_{n}<h(t)+u\right\} .
\end{aligned}
$$

From the first equality (9), it follows from the law of total probability that

$$
\mathbb{P}\left(\tau_{u, b}>t\right)=\sum_{n=0}^{+\infty} \mathbb{P}\left(\tau_{u, b}>t \mid N(t)=n\right) \mathbb{P}[N(t)=n] .
$$


By considering the possible values for the sequence of partial sums $\left\{S_{n}, n \geq 1\right\}$, the conditional probability in $(10)$ is written as

$$
\begin{aligned}
\mathbb{P}\left(\tau_{u, b} \geq t \mid N(t)=n\right)= & \mathbb{E}\left(\mathbb { P } \left\{\bigcap_{k=1}^{n}\left\{h^{-1}\left[\left(S_{k}-u\right)_{+}\right] \leq T_{k} \leq h^{-1}\left(S_{k-1}+b-u\right)\right\}\right.\right. \\
& \left.\mid N(t)=n\} \mathbb{I}_{\left\{h(t)-(b-u)<S_{n}<h(t)+u\right\}}\right)
\end{aligned}
$$

and by the order statistic property, it follows that

$$
\begin{aligned}
& \mathbb{P}\left\{\bigcap_{k=1}^{n}\left\{h^{-1}\left[\left(S_{k}-u\right)_{+}\right] \leq T_{k} \leq h^{-1}\left(S_{k-1}+b-u\right)\right\} \mid N(t)=n\right\} \\
= & \mathbb{P}\left\{\bigcap_{k=1}^{n}\left\{h^{-1}\left[\left(S_{k}-u\right)_{+}\right] \leq V_{k: n} \leq h^{-1}\left(S_{k-1}+b-u\right)\right\}\right\},
\end{aligned}
$$

where $\left(V_{1: n}, \ldots, V_{1: n}\right)$ are the order statistics for $n$ i.i.d. random variables of distribution function $F_{t}$. Since

$$
F_{t}\left(V_{k: n}\right) \stackrel{d}{=} U_{k: n}, 1 \leq k \leq n,
$$

where $\left(U_{1: n}, \ldots, U_{n: n}\right)$ are the order statistics of a sample of $n$ standard uniform random variables, the conditionnal probability in (11) may be rewritten as

$$
\begin{aligned}
& \mathbb{P}\left\{\bigcap_{k=1}^{n}\left\{h^{-1}\left[\left(S_{k}-u\right)_{+}\right] \leq V_{k: n} \leq h^{-1}\left(S_{k-1}+b-u\right)\right\}\right\} \\
= & \mathbb{P}\left(\bigcap_{k=1}^{n}\left\{F_{t}\left\{h^{-1}\left[\left(S_{k}-u\right)_{+}\right]\right\} \leq U_{k: n} \leq F_{t}\left[h^{-1}\left(S_{k-1}+b-u\right)\right]\right\}\right) \\
= & d_{n}\left(\left\{\left(F_{t}\left\{h^{-1}\left[\left(S_{k}-u\right)_{+}\right]\right\}, F_{t}\left[h^{-1}\left(S_{k-1}+b-u\right)\right]\right) ; 1 \leq k \leq n\right\}\right) .
\end{aligned}
$$

Combining (10), (11), and (12) yields the result (8).

The numerical evaluation of the two-sided ruin probability in $(8)$ is not an easy task. The explicit form involves an infinite serie and multiple integrals. A truncation and numerical integration, consolidated by techniques developped by Dimitrova et al. 8 is one option to compute $\phi(u, b, t)$. An alternative approach will be presented in a forthcoming paper.

\section{The density of the win-first time $\tau_{u, b}^{*}$}

In this section, the study of the ordered risk process continues. From now on the function $h$ is assumed to be continuous and differentiable. Additionnaly, the claim sizes form an i.i.d. sequence of non-negative random variables with $\mathrm{PDF} f_{U}$. The next result proposes a formula for the distribution of the stopping time

$$
\tau_{u, b}^{*}=\inf \{t \geq 0, R(t) \geq b \text { and } R(s) \geq 0 \text { for } s \leq t \mid R(0)=u\} .
$$

The expression involves the PDF of the compound process $\left\{S_{N(t)}, t \geq 0\right\}$. Because the claim sizes are non-negative i.i.d. random variables then the PDF of $S_{N(t)}$ is denoted by $f_{U}^{* N(t)}$ and corresponds to the $N(t)^{t h}$ convolution of the PDF of $U$ with itself. 
Theorem 1. The win-first-time $\tau_{u, b}^{*}$ takes values $t \geq h^{-1}(b-u)$. It has an atom at $h^{-1}(b-u)$ with

$$
\mathbb{P}\left[\tau_{u, b}^{*}=h^{-1}(b-u)\right]=\mathbb{P}\left\{N\left[h^{-1}(b-u)\right]=0\right\},
$$

while for $t>h^{-1}(b-u)$ it has a density part given by

$$
f_{\tau_{u, b}^{*}}(t)=\mathbb{E}\left\{g_{N(t)}(t, u, b) h^{(1)}(t) f_{U}^{* N(t)}[h(t)-(b-u)] \mathbb{1}_{\{N(t)>0\}}\right\},
$$

where

$$
g_{n}(t, u, b)=\mathbb{E}^{*}\left[d_{n}\left(\left\{\left(F_{t}\left\{h^{-1}\left[\left(S_{k}-u\right)_{+}\right]\right\}, F_{t}\left[h^{-1}\left(S_{k-1}+b-u\right)\right]\right) ; 1 \leq k \leq n\right\}\right)\right] .
$$

The expectation $E^{*}$ is the conditional expectation with respect to the event $\left\{S_{n}=h(t)-\right.$ $(b-u)\}$, the function $h^{(1)}(t)$ denotes the first order derivative of $h(t)$, and $d_{n}(\{\ldots\})$ are the rectangular probabilities as defined in the Section 2 .

Proof. Clearly, the risk process $\{R(t), t \geq 0\}$ cannot reach the level $b$ before time $h^{-1}(b-$ $u)$. However, if no claim is filed until time $h^{-1}(b-u)$, which happens with probability $\mathbb{P}\left\{N\left[h^{-1}(b-u)\right]=0\right\}$, then the risk process hits the level $b$ hence (14). Let me now introduce two stopping times. The first one is the classical ruin time defined by

$$
\tau_{u}=\inf \{t \geq 0 ; R(t)<0 \mid R(0)=u\},
$$

and rewritten as

$$
\begin{aligned}
\tau_{u} & =\inf \left\{t \geq 0 ; u+h(t)-S_{N(t)}<0\right\} \\
& =\inf \left\{t \geq 0 ; S_{N(t)}>u+h(t)\right\} .
\end{aligned}
$$

Thus the ruin time reduces to the first-crossing time of the stochastic process $\left\{S_{N(t)} ; t \geq\right.$ $0\}$ through the upper boundary $u+h(t)$. Note that the event $\left\{\tau_{u}>t\right\}$ is equivalent to

$$
\begin{aligned}
\left\{\tau_{u}>t\right\} & =\bigcup_{n=0}^{+\infty}\{N(t)=n\} \cap\left\{\tau_{u}>t\right\} \\
& =\bigcup_{n=0}^{+\infty}\{N(t)=n\} \bigcap_{k=1}^{n}\left\{T_{k} \geq h^{-1}\left[\left(S_{k}-u\right)_{+}\right]\right\} \cap\left\{S_{n} \leq u+h(t)\right\} .
\end{aligned}
$$

A second stopping time is defined as

$$
\tau_{b}^{*}=\inf \{t \geq 0 ; R(t) \geq b\},
$$

and corresponds to the first time at which the risk process reaches the level $b \geq u$. The first-meeting time $(18)$ is rewritten as

$$
\begin{aligned}
\tau_{b}^{*} & =\inf \left\{t \geq 0 ; u+h(t)-S_{N(t)} \geq b\right\} \\
& =\inf \left\{t \geq 0 ; S_{N(t)} \leq h(t)-(b-u)\right\}
\end{aligned}
$$


and thus is interpreted as the first time at which the stochastic process $\left\{S_{N(t)} ; t \geq 0\right\}$ meets the lower boundary $h(t)-(b-u)$. Consider the event $\left\{\tau_{b}^{*} \in[t, t+\mathrm{d} t]\right\}$, for $t>h^{-1}(b-u)$ and $\mathrm{d} t$ small enough, it follows that

$$
\begin{aligned}
\left\{\tau_{b}^{*} \in[t, t+\mathrm{d} t\}=\right. & \bigcup_{n=1}^{+\infty}\{N(t)=n\} \cap\left\{\tau_{b}^{*} \in[t, t+\mathrm{d} t]\right\} \\
= & \bigcup_{n=1}^{+\infty}\{N(t)=n\} \bigcap_{k=1}^{n}\left\{T_{k} \leq h^{-1}\left[S_{k-1}-(b-u)\right]\right\} \\
& \bigcap\left\{h^{-1}\left[S_{n}-(b-u)\right] \in[t, t+\mathrm{d} t]\right\} .
\end{aligned}
$$

Note that at least one claim has been filed, otherwise the level $b$ was reached at time $h^{-1}(b-u)$. Next, consider the event $\left\{\tau_{u, b}^{*} \in[t, t+\mathrm{d} t]\right\}$. Combining (17) and (19) yields

$$
\begin{aligned}
\left\{\tau_{u, b}^{*} \in[t, t+\mathrm{d} t\}=\right. & \left\{\tau_{u} \geq t\right\} \cap\left\{\tau_{b}^{*} \in[t, t+\mathrm{d} t]\right\} \\
= & \bigcup_{n=1}^{+\infty}\{N(t)=n\} \bigcap_{k=1}^{n}\left\{h^{-1}\left[\left(S_{k}-u\right)_{+}\right] \leq T_{k} \leq h^{-1}\left[S_{k-1}-(b-u)\right]\right\} \\
& \bigcap\left\{h^{-1}\left[S_{n}-(b-u)\right] \in[t, t+\mathrm{d} t]\right\} .
\end{aligned}
$$

Using the law of total probability yields

$$
\mathbb{P}\left(\tau_{u, b}^{*} \in[t, t+\mathrm{d} t]\right)=\sum_{n=1}^{+\infty} \mathbb{P}\left[\tau_{u, b}^{*} \in[t, t+\mathrm{d} t] \mid N(t)=n\right] \mathbb{P}[N(t)=n],
$$

and from $(21)$, the conditional expectation in $(22)$, becomes

$$
\begin{aligned}
\mathbb{P}\left[\tau_{u, b}^{*} \in[t, t+\mathrm{d} t] \mid N(t)=n\right]= & \mathbb{P}\left[\bigcap_{k=1}^{n}\left\{h^{-1}\left[\left(S_{k}-u\right)_{+}\right] \leq T_{k} \leq h^{-1}\left[S_{k-1}-(b-u)\right]\right\}\right. \\
& \left.\bigcap\left\{h^{-1}\left[S_{n}-(b-u)\right] \in[t, t+\mathrm{d} t]\right\} \mid N(t)=n\right]
\end{aligned}
$$

By the order statistics property, given that $[N(t)=n](n \geq 1)$, the vector $\left(T_{1}, \ldots, T_{n}\right)$ is distributed as the order statistics $\left(V_{1: n}, \ldots, V_{n: n}\right)$ of a sample of $n$ i.i.d. random variables with distribution function $F_{t}(s)$, for $s \leq t$. This implies that

$$
\left[F_{t}\left(V_{1: n}\right), \ldots, F_{t}\left(V_{n: n}\right)\right] \stackrel{\mathcal{D}}{=}\left(U_{1: n}, \ldots, U_{n: n}\right),
$$

where $\left(U_{1: n}, \ldots, U_{n: n}\right)$ are the order statistics of $n$ i.i.d. uniform random variables on $(0,1)$. Thanks to 24 , the conditional probability $(23)$ can be rewritten as

$$
\begin{aligned}
& \mathbb{P}\left[\left\{\tau_{u, b}^{*} \in[t, t+\mathrm{d} t\} \mid N(t)=n\right]\right. \\
= & \mathbb{P}\left[\bigcap_{k=1}^{n}\left\{h^{-1}\left[\left(S_{k}-u\right)_{+}\right] \leq T_{k} \leq h^{-1}\left[S_{k-1}-(b-u)\right]\right\}\right. \\
& \left.\bigcap\left\{h^{-1}\left[S_{n}-(b-u)\right] \in[t, t+\mathrm{d} t]\right\} \mid N(t)=n\right]
\end{aligned}
$$




$$
\begin{aligned}
= & \mathbb{P}\left[\bigcap_{k=1}^{n}\left\{h^{-1}\left[\left(S_{k}-u\right)_{+}\right] \leq V_{k: n} \leq h^{-1}\left[S_{k-1}-(b-u)\right]\right\}\right. \\
& \left.\bigcap\left\{h^{-1}\left[S_{n}-(b-u)\right] \in[t, t+\mathrm{d} t]\right\}\right] \\
= & \mathbb{P}\left[\bigcap_{k=1}^{n}\left\{F_{t}\left\{h^{-1}\left[\left(S_{k}-u\right)_{+}\right]\right\} \leq U_{k: n} \leq F_{t}\left\{h^{-1}\left[S_{k-1}-(b-u)\right]\right\}\right\}\right. \\
& \left.\mid\left\{h^{-1}\left[S_{n}-(b-u)\right] \in[t, t+\mathrm{d} t]\right\}\right] \mathbb{P}\left[\left\{h^{-1}\left[S_{n}-(b-u)\right] \in[t, t+\mathrm{d} t]\right\}\right] .
\end{aligned}
$$

Using the notation introduced in Section 2 for the rectangular probabilities of the order statistics yields

$$
\begin{aligned}
& \mathbb{P}\left[\bigcap_{k=1}^{n}\left\{F_{t}\left\{h^{-1}\left[\left(S_{k}-u\right)_{+}\right]\right\} \leq U_{k: n} \leq F_{t}\left\{h^{-1}\left[S_{k-1}-(b-u)\right]\right\}\right\}\right. \\
& \left.\mid\left\{h^{-1}\left[S_{n}-(b-u)\right] \in[t, t+\mathrm{d} t]\right\}\right] \\
= & \mathbb{E}\left\{\mathbb { P } \left[\bigcap_{k=1}^{n}\left\{F_{t}\left\{h^{-1}\left[\left(S_{k}-u\right)_{+}\right]\right\} \leq U_{k: n} \leq F_{t}\left\{h^{-1}\left[S_{k-1}-(b-u)\right]\right\}\right\}\right.\right. \\
= & \left.\left.\mid S_{0}, \ldots S_{n}\right] \mid S_{n}=h(t)-(b-u)\right\} \\
& \left(F _ { t } \left\{h ^ { - 1 } \left[\left(F_{t}\left\{h^{-1}\left[\left(S_{1}-u\right)_{+}\right]\right\}, F_{t}\left\{h^{-1}\left[S_{0}-(b-u)\right]\right\}\right), \ldots,\right.\right.\right. \\
= & g_{n}(t, u, b), n \geq 0 .
\end{aligned}
$$

On the other hand, note that

$$
\mathbb{P}\left[\left\{h^{-1}\left[S_{n}-(b-u)\right] \in[t, t+\mathrm{d} t]\right\}\right]=h^{(1)}(t) f_{U}^{* n}[h(t)-(b-u)] \mathrm{d} t, t \geq 0 .
$$

Inserting $(26)$ and $(27)$ into 22 yields the result (15).

As for the survival function given in Proposition 3, a numerical evaluation of the defective PDF in (15) can be achieved via truncation and numerical integration, as in, for example, the paper of Borovkov and Dickson [2].

\section{Concluding remarks}

This paper provides a formula for the survival function of the first-exit time of the risk process from a region delimited by two horizontal barriers. A formula to study the distribution of the first-exit time of the risk process through the upper barrier is also derived. Both derivations involve the joint distribution of the order statistics of a sample of uniform random variables. The numerical evaluation represents an interesting challenge. A procedure relying on the truncation of the infinite series followed by numerical integration may be utilized. An alternative method is currently under construction and will be presented and compared to the numerical integration routine in a future research work. This work is a natural extension of the one presented in Goffard and Lefèvre [10, Section 5], as the stochastic process is allowed to perform upward jumps of random size. It bridges 
the study of the crossing of the classical ruin barrier proposed in Lefèvre and Picard [12] and the meeting of the upper barrier as in Picard and Lefèvre [18]. The occurrence of remarkable families of polynomials connects the two aforementioned works and is partly explained by the probabilistic interpretation of those polynomials as function linked to the joint distribution of uniform order statistics.

\section{Acknowledgments}

This work has been done while the author was visiting the Department of Statistics and Applied Probability of the University of California, Santa Barbara. The author is thankful for the warm welcome coming from the members of the PSTAT department of UCSB. This work was partially funded by a CAE grant of the Society of Actuaries.

\section{References}

[1] B. Avanzi. Strategies for dividend distribution: A review. North American Actuarial Journal, 13(2):217-251, 2009.

[2] K. A. Borovkov and D. C. M. Dickson. On the ruin time distribution for a Sparre Andersen process with exponential claim sizes. Insurance: Mathematics and Economics, 42(3):1104-1108, 2008.

[3] K. S. Crump. On point processes having an order statistic structure. Sankhyā: The Indian Journal of Statistics, Series A (1961-2002), 37(3):396-404, 1975.

[4] M. Denuit, C. Lefèvre, and P. Picard. Polynomial structures in order statistics distributions. Journal of Statistical Planning and Inference, 113(1):151-178, 2003.

[5] D. C. M. Dickson and J. R. Gray. Approximations to ruin probability in the presence of an upper absorbing barrier. Scandinavian Actuarial Journal, 1984(2):105-115, 1984.

[6] D. S. Dimitrova, Z. G. Ignatov, and V. K. Kaishev. Ruin and deficit under claim arrivals with the order statistics property. working paper or preprint, 2016.

[7] D. S. Dimitrova, Z. G. Ignatov, and V. K. Kaishev. On the first crossing of two boundaries by an order statistics risk process. Risks, 5(3):43, 2017.

[8] D.S. Dimitrova, V. K. Kaishev, and S. Zhao. On the evaluation of finite-time ruin probabilities in a dependent risk model. Applied Mathematics and Computation, 275(Supplement C):268-286, 2016.

[9] P.-O. Goffard and C. Lefèvre. Duality in ruin problems for ordered risk models. working paper or preprint, 2016.

[10] P.-O. Goffard and C. Lefèvre. Boundary crossing of order statistics point processes. Journal of Mathematical Analysis and Applications, 447(2):890-907, 2017. 
[11] Z. G. Ignatov and V. K. Kaishev. First crossing time, overshoot and appellhessenberg type functions. Stochastics, 88(8):1240-1260, 2016.

[12] C. Lefèvre and P. Picard. A new look at the homogeneous risk model. Insurance: Mathematics and Economics, 49(3):512-519, 2011.

[13] C. Lefèvre and P. Picard. Ruin probabilities for risk models with ordered claim arrivals. Methodology and Computing in Applied Probability, 16(4):885-905, 2014.

[14] C. Lefèvre and P. Picard. Risk models in insurance and epidemics: A bridge through randomized polynomials. Probability in the Engineering and Informational Sciences, $29(3): 399-420,2015$.

[15] A. Lehmann. Boundary crossing probabilities of Poisson counting processes with general boundaries. Advances in Stochastic Models for Reliability, Quality and Safety, pages 153-166, 1998.

[16] H. Niederhausen. Sheffer polynomials for computing exact Kolmogorov-Smirnov and Rényi distributions. The Annals of Statistics, 9(5):923-944, 1981.

[17] D. Perry, W. Stadje, and S. Zacks. A two-sided first-exit problem for a compound Poisson process with a random upper boundary. Methodology and Computing in Applied Probability, 7(1):51-62, 2005.

[18] P. Picard and C. Lefèvre. On the first crossing of the surplus process with a given upper barrier. Insurance: Mathematics and Economics, 14(2):163-179, 1994.

[19] P. S. Puri. On the characterization of point processes with the order statistic property without the moment condition. Journal of Applied Probability, 19(1):39-51, 1982.

[20] D. Rullière and S. Loisel. The win-first probability under interest force. Insurance: Mathematics and Economics, 37(3):421-442, 2005.

[21] F. E. De Vylder and M. J. Goovaerts. Inequality extensions of Prabhu's formula in ruin theory. Insurance: Mathematics and Economics, 24(3):249-271, 1999.

[22] F. E. De Vylder and M. J. Goovaerts. Homogeneous risk models with equalized claim amounts. Insurance: Mathematics and Economics, 26(2-3):223-238, 2000.

[23] G. E. Willmot. The total claims distribution under inflationary conditions. Scandinavian Actuarial Journal, 1989(1):1-12, 1989.

[24] Y. Xu. First exit times of compound Poisson processes with parallel boundaries. Sequential Analysis, 31(2):135-144, 2012. 